Gut and Liver, Vol. 10, No. 6, November 2016, pp. 896-901

\title{
Impact of Long-Term Proton Pump Inhibitor Therapy on Gut Microbiota in F344 Rats: Pilot Study
}

\author{
Cheol Min Shin ${ }^{1}$, Nayoung $\mathrm{Kim}^{1,2}$, Yong Sung $\mathrm{Kim}^{3}$, Ryoung Hee Nam${ }^{1}$, Ji Hyun Park ${ }^{2}$, Dong Ho Lee ${ }^{1,2}$, Yeong-Jae Seok ${ }^{4}$, \\ Yeon-Ran Kim ${ }^{4}$, Joo-Hyon Kim ${ }^{5}$, Jung Min Kim ${ }^{6}$, Joo Sung $\mathrm{Kim}^{2}$, and Hyun Chae Jung ${ }^{2}$ \\ ${ }^{\prime}$ Department of Internal Medicine, Seoul National University Bundang Hospital, Seongnam, ${ }^{2}$ Department of Internal Medicine and Liver \\ Research Institute, Seoul National University College of Medicine, Seoul, ${ }^{3}$ Gastroenterology and Digestive Disease Research Institute, \\ Wonkwang University School of Medicine, Iksan, ${ }^{4}$ Department of Biological Sciences and Institute of Microbiology, Seoul National University, \\ Seoul, ${ }^{5}$ Life Science R\&D Center, SK Chemicals, Seongnam, and ${ }^{6}$ NAR Center, Inc., Daejeon Oriental Hospital of Daejeon University, Daejeon, \\ Korea
}

See editorial on page 865.

Background/Aims: To evaluate changes in gut microbiota composition following long-term proton pump inhibitor (PPI) treatment. Methods: Twenty-four-week-old F344 rats were fed diets with $(n=6)$ or without $(n=5)$ lansoprazole for 50 weeks. Profiles of luminal microbiota in the terminal ileum were then analyzed. Pyrosequencing of the $16 \mathrm{~S}$ rRNA gene was performed using an FLX genome sequencer (454 Life Sciences/Roche). Results: Rats treated with lansoprazole showed significantly reduced body weights compared to controls (lansoprazole-treated rats and controls, 322.3 \pm 15.3 g vs $403.2 \pm 5.2 \mathrm{~g}$, respectively, $\mathrm{p}<0.001$ ). However, stool frequencies and consistencies did not differ between the two groups. The composition of the gut microbiota in lansoprazole-treated rats was quite different from that of the controls. In the controls, the microbiota profiles obtained from the terminal ileum showed a predominance of Proteobacteria (93.9\%) due to the abundance of Escherichia and Pasteurella genera. Conversely, lansoprazole-treated rats showed an elevated population of Firmicutes (66.9\%), which was attributed to an increased ratio of Clostridium g4 to Lactobacillus genera. Conclusions: This preliminary study suggests that long-term administration of PPI may cause weight loss and changes to the microbiota in the terminal ileum. (Gut Liver 2016;10:896-901)

Key Words: Proton pump inhibitors; Gastrointestinal microbiome; Small bowel intestinal overgrowth; Clostridium

\section{INTRODUCTION}

Proton pump inhibitors (PPIs) are frequently prescribed by primary care physicians and gastroenterologists for a wide range of indications, including treatment of peptic ulcer disease, gastroesophageal reflux disease, and as part of the treatment regimen for Helicobacter pylori.

The long-term use of PPIs is increasing. ${ }^{1,2}$ This prolonged use may feature side effects or complications of PPI therapy, such as bone metabolism, fracture, hypomagnesaemia, vitamin $B_{12}$ deficiency, iron deficiency anemia, and pneumonia. ${ }^{3-5}$ In addition, an increasing number of studies have evaluated whether the long-term use of PPI causes small intestinal bacterial overgrowth (SIBO) or Clostridium difficile infection. However, conflicting results have prevented any conclusion of the risk of SIBO among PPI users. ${ }^{6,7}$

Chronic acid suppression and resulting hypochlorhydria associated with PPI use may alter the intraluminal environment to promote growth of the bacterial flora in the small intestine. ${ }^{8}$ In addition, a change in proximal intestinal $\mathrm{pH}$ might alter the gut microbiota. ${ }^{9}$ This could subsequently affect human health including weight loss. However, evidence is scant.

This preliminary study assessed the changes in gut microbiota composition by long-term PPI treatment using F344 rats in relation to weight loss.

\section{MATERIALS AND METHODS}

\section{Animals and tissue preparation}

Specific pathogen-free, 344, male Fischer (F344) rats were

Correspondence to: Nayoung Kim

Department of Internal Medicine, Seoul National University Bundang Hospital, 82 Gumi-ro 173beon-gil, Bundang-gu, Seongnam 13620, Korea

Tel: +82-31-787-7008, Fax: +82-31-787-4051, E-mail: nayoungkim49@empas.com

Received on October 21, 2015. Revised on January 17, 2016. Accepted on February 1, 2016. Published online July 27, 2016

pISSN 1976-2283 eISSN 2005-1212 https://doi.org/10.5009/gnl15529

@ This is an Open Access article distributed under the terms of the Creative Commons Attribution Non-Commercial License (http://creativecommons.org/licenses/by-nc/4.0) which permits unrestricted non-commercial use, distribution, and reproduction in any medium, provided the original work is properly cited. 
used (Orient Bio, Seongnam, Korea). Each animal was housed in a cage maintained at $23^{\circ} \mathrm{C}$ with alternating 12-hour cycles of light and dark under specific pathogen-free conditions. Beginning at 24 weeks of age, a control group of five rats was fed ad libitum with Purina rat chow for 50 weeks. Another group of six rats received the same feed supplemented with lansoprazole ( $5 \mathrm{mg} / \mathrm{kg} /$ day). The rats were sacrificed after the 50 -week period. In 24-week old rat, average body weight and daily chow intake were presumed to be $400 \mathrm{~g}$ and $40 \mathrm{~g}$, respectively. One gram lansoprazole was mixed with $20 \mathrm{~kg}$ rat chow to obtain the daily dose. A recent study from our group of the long-term (1.5 years) administration of lansoprazole $5 \mathrm{mg} / \mathrm{kg} /$ day found that 74 -week old F344 rats in the lansoprazole group had lower stimulated gastric acid secretion than those in the vehicle group. ${ }^{10}$ In the present study, the rats were fasted but allowed water for 12 hours before the experiments. The animals were anesthetized by zoletil and rompun mixture and killed by decapitation. A 1-cm length of the terminal ileum per rat was obtained and phosphate-buffered saline was flushed through the lumens. The flushed material was centrifuged at 20,000×g for 30 minutes to pellet bacteria. The pellet was processed to extract total microbial DNA using a commercial kit (iNtRON Biotechnology, Seongnam, Korea). This study was approved by the Institutional Animal Care and Use Committee (IACUC) of Seoul National University Bundang Hospital (IACUC number: BA0903-040/01301).

\section{Barcoded 454-pyrosequencing of the 16S rRNA gene}

DNA samples from 11 74-week-old F344 rats were subjected to pyrosequencing. Polymerase chain reaction (PCR) amplification was performed using primers targeting the V1 to V3 regions of the 16S rRNA gene with extracted DNA. For bacterial amplification, barcoded primers were 9F (5'-CCTATCCCCTGTGTGCCTTGGCAGTC-TCAG-AC-AGAGTTTGATCMTGGCTCAG-3'; underlined sequence indicates the target region primer) and 541R (5'-CCATCTCATCCCTGCGTGTCTCCGAC-TCAG-X-ACATTACCGCGGCTGCTGG-3'; ' $\mathrm{X}$ ' indicates the unique barcode for each subject) (http://oklbb.ezbiocloud.net/content/1001). Amplifications were carried out using an initial denaturation at $95^{\circ} \mathrm{C}$ for 5 minutes, followed by 30 cycles of denaturation at $94^{\circ} \mathrm{C}$ for 30 seconds, primer annealing at $55^{\circ} \mathrm{C}$ for 45 seconds, and extension at $72^{\circ} \mathrm{C}$ for 90 seconds, with a final elongation at $72^{\circ} \mathrm{C}$ for 5 minutes. PCR products were analyzed by $2 \%$ agarose gel electrophoresis and visualized using a Gel Doc system (BioRad, Hercules, CA, USA). The amplified products were purified with the QIAquick PCR purification kit (Qiagen, Valencia, CA, USA). Equal concentrations of purified products were pooled together, and short fragments (nontarget products) were removed with Ampure beads kit (Agencourt Bioscience, Beverly, MA, USA). The quality and product size were assessed on a Bioanalyzer 2100 (Agilent Technologies, Palo Alto, CA, USA) using a DNA 7500 chip. Mixed amplicons were subjected to emulsion
PCR, and then deposited on picotiter plates. The sequencing was carried out at ChunLab, Inc. (Seoul, Korea) with GS Junior Sequencing system, the modified laboratory benchtop form of 454 sequencing systems (Roche, Branford, CT, USA) according to the manufacturer's instructions. The raw unprocessed 16S rRNA gene sequence which had been generated by our study was deposited in National Center for Biotechnology Information's Sequence Read Archive (NCBI's SRA) (Gene Expression Omnibus [GEO] accession: GSE 67800).

\section{Pyrosequencing data analysis}

Reads obtained from different samples were sorted by unique barcodes of each PCR product. The barcode, linker, and primer sequences were removed from the original sequencing reads. Reads containing two or more ambiguous nucleotides, reads with a low quality score (average score <25), or reads shorter than $300 \mathrm{bp}$, were discarded. Potential chimeric sequences were detected by the Bellerophon method, which compares the BLASTN search results between the forward half and reverse half sequences. ${ }^{11}$ After removing the chimeric sequences, the taxonomic classification of each read was assigned against the EzTaxon-e database (http://eztaxon-e.ezbiocloud.net), ${ }^{12}$ which contains the 16S rRNA gene sequence of type strains that have valid published names and representative species level phylotypes of either cultured or uncultured entries in the GenBank database with complete hierarchical taxonomic classification from the phylum to the species.

The richness and diversity of samples were determined by ACE, Chao1 and Jackknife estimation. In addition, Simpson diversity index and Shannon diversity index at 3\% distance was calculated in the CLcommunity program (ChunLab Inc.). Good's method was used to calculate sequencing coverage. ${ }^{13}$

Random subsampling was conducted to equalize the read size of samples for comparing different read sizes among samples. To compare the operational taxonomic units (OTUs) between samples, shared OTUs were obtained with the XOR analysis of the CLcommunity program. UniFrac-based principal coordinates analysis was used to compare the microbial communities of subgroups.

\section{Statistical analyses}

Statistical calculations other than pyrosequencing data were performed using SPSS version 18.0 (SPSS Inc., Chicago, IL, USA). The two groups were compared by the Student t-test or Mann-Whitney U test and p-values $<0.05$ were considered as statistically significant

\section{RESULTS}

\section{Body weight and bowel habit changes in PPI-treated rats}

Rats treated with lansoprazole showed significantly reduced body weight compared to controls $(322.3 \pm 15.3 \mathrm{~g}$ vs $403.2 \pm 5.2$ 
$\mathrm{g}, \mathrm{p}<0.001)$. However, stool frequency and consistency were not different between the two groups.

\section{Pyrosequencing result of terminal ileum luminal samples: changes of gut microbiota in PPI-treated rats}

Total microbial DNAs from 11 terminal ileum luminal samples were analyzed by pyrosequencing. A total of 48,794 16S rRNA reads were obtained. The composition of the gut microbiota in lansoprazole-treated rats was markedly different from that in controls. The major phylum in the ileum luminal microbiota was Proteobacteria in controls and Firmicutes in lansoprazole-treated rats (Fig. 1). An Unweighted Pair Group Method with Arithmetic mean dendrogram indicated genetic relationships of the samples, which identified four distinct groups (I, II, III, and IV) (Fig. 2). In control rats (group I), microbiota profiles

\section{A}

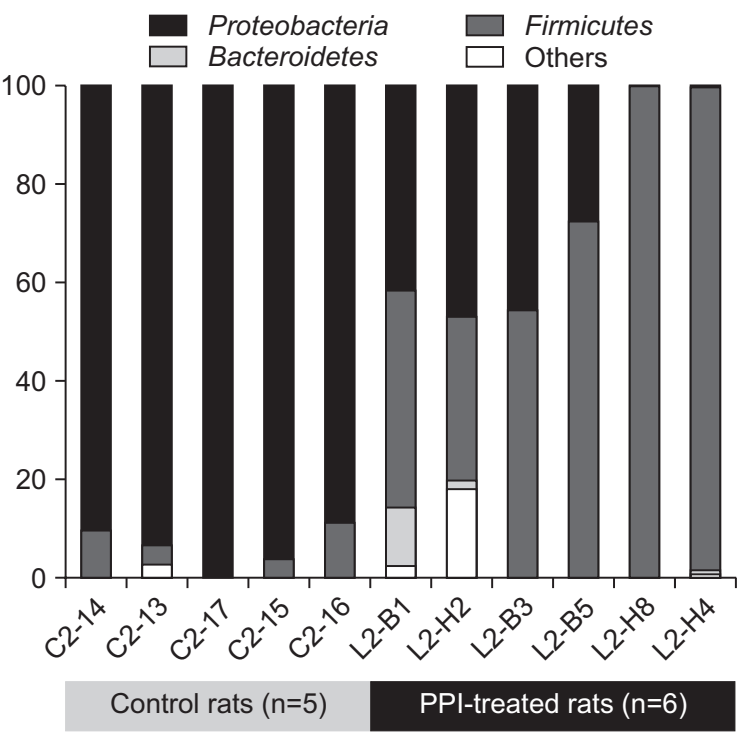

\section{B}

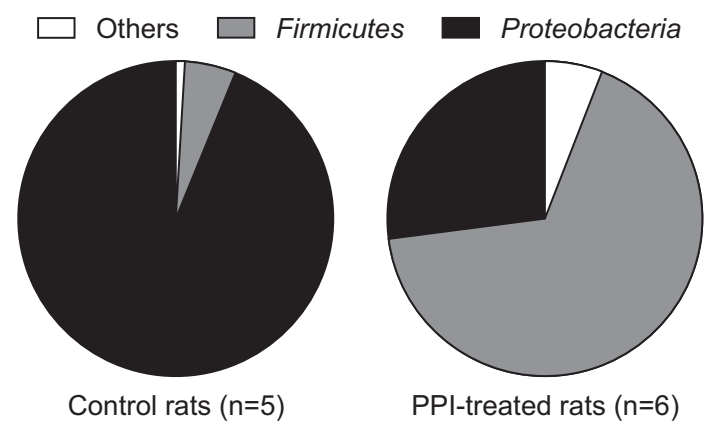

Fig. 1. Distribution of the phyla in different samples from the terminal ileum. (A) C2-13, C2-14, C2-15, C2-16 and C2-17 belong to the control group, and L2-B1, L2-B3, L2-B5, L2-H2, L2-H4, L2-H8 belong to the proton pump inhibitor (PPI)-treated group. (B) The composition of the gut microbiota in PPI-treated rats was quite different from that of the controls. The major phyla were Proteobacteria in the controls and Firmicutes in the PPI-treated rats. obtained from terminal ileum were homogenous, which showed a predominance of Proteobacteria (93.9\%) due to increased Escherichia or Pasteurella genera (71.5\% and 20.6\%, respectively). Although Firmicutes (66.9\%) was the major phylum in lansoprazole-treated rats, the composition of bacterial community in PPI-treat rats appeared to be heterogeneous and could be categorized into groups II, III, IV. Especially, in group III, the abundance of the Firmicutes was attributed to an increased ratio of Clostridium g4 genus (74.1\%); in group IV, it resulted from the increase of Lactobacillus genus (80.8\%) (Fig. 3).

With regard to the diversity of the bacterial community, the average number of reads per specimen was 4,435.8 $\pm 1,852.2$ and the overall Good's coverage was 98\%. The average number of OUT counts was not different between the PPI and control groups ( $p>0.05$, Student t-test.) Species richness indicators including ACE, Chao1, and Jackknife, were calculated by the CLcommunity program, but there was no significant difference in ACE, Chao1, or Jackknife between the two groups. Shannon diversity and Simpson indices were also statistically insignificant ( $p>0.05$ ). OTU counts, richness indicators, and diversity indices in each individual animal are presented in Supplementary Table 1.

\section{DISCUSSION}

Chronic administration of PPI was associated with weight loss and alternation of terminal ileal luminal microbiota in F344 rats. A few previous studies investigated the changes of gut microbiota after PPI use, but most evaluated short-term effects only. Some of our findings were consistent with these studies, but some were not. Several aspects should be considered to interpret the discrepancy between the studies.

The effect of PPI on $\mathrm{pH}$ is limited to the stomach and proximal duodenum, with little or no effects on the $\mathrm{pH}$ of distal gut. ${ }^{14}$

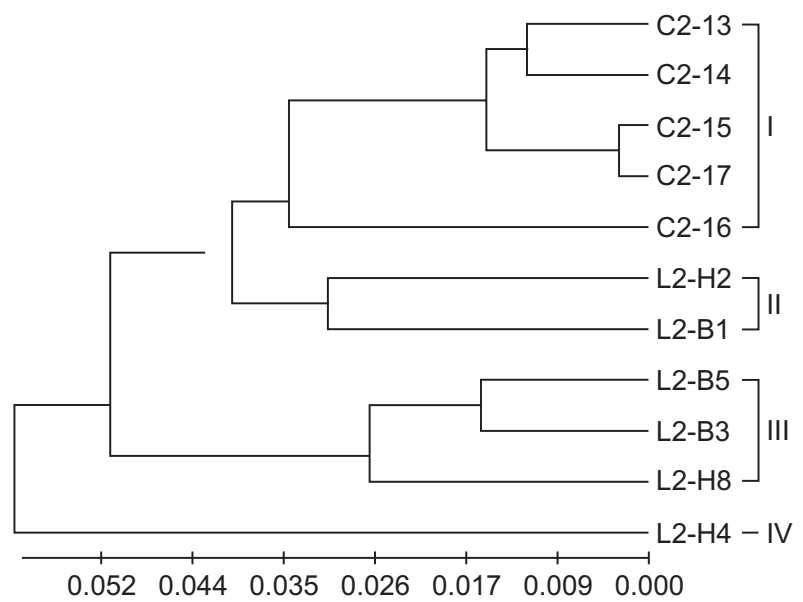

Fig. 2. Fast UniFrac analysis for the comparison of the microbial communities (unweighted pair group method with arithmetic mean dendrogram) that identified four distinct groups (I, II, III, and IV). 


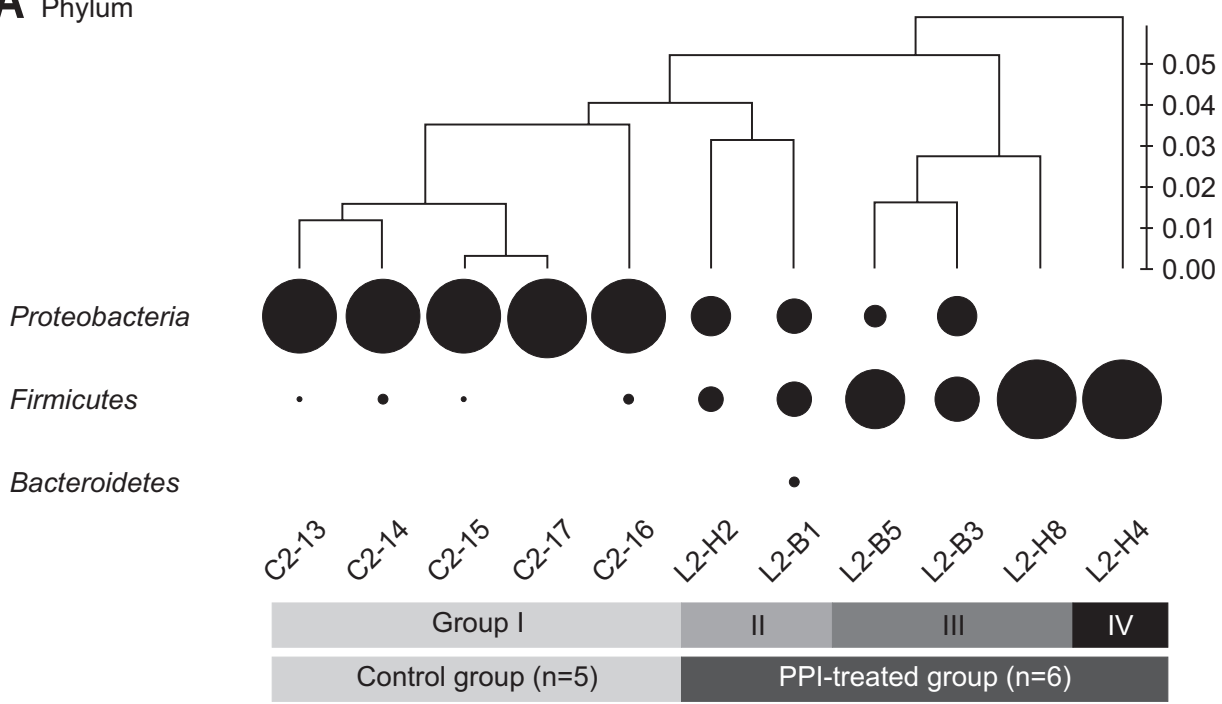

Bacteroidetes

B

Genus

Escherichia

Clostridium g4

Pasteurella g2

Lactobacillus

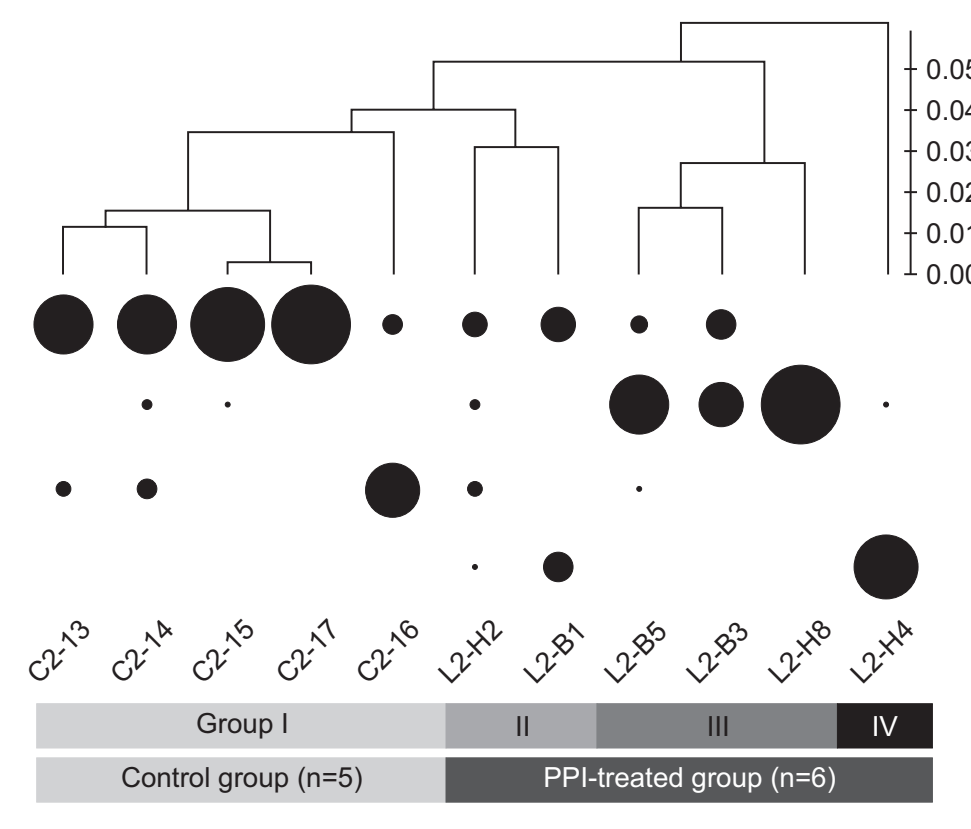

Fig. 3. Circular heat map analysis of phyla (A) and genera (B). Each taxon and its proportion are represented by a circle (area), with a minimum ratio for inclusion of 5\%.

PPI, proton pump inhibitor.
However, gastric acid suppression by PPI may affect intestinal bacterial composition irrespective of intestinal pH. ${ }^{7}$ Notably, gastric acid secretion is affected both by PPI and by aging. Gastric hypochlorhydria in the elderly has been associated with the risk of developing SIBO. ${ }^{15,16}$ Recently, we evaluated gastric acid secretion in the male F344 rats of four different age groups (6-, 31-, and 74-week, and 2-year) and found that both basal and stimulated gastric acid secretions significantly decreased with increased connective tissue of lamina propria with age. ${ }^{10}$ Nevertheless, we observed that lansoprazole decreased stimulated gastric acid secretion in 74-week-old F344 rats. ${ }^{10}$ This indicates that PPI is also effective suppressing gastric acid secretion in elderly rats. In addition, the intestinal microbiota may change with age. One study reported that the ratio of Firmicutes to Bacteroidetes ( $\mathrm{F} / \mathrm{B}$ ratio) in human feces evolves during different life stages; the F/B ratio for infants, adults and elderly individuals was 0.4 , 10.9 , and 0.6, respectively. ${ }^{17}$

In this study, luminal microbiota of terminal ileum was evaluated. We hypothesized that long-term administration of PPI is associated with both SIBO and C. difficile infection. Changes of small intestinal microbiota composition are known to be prominent in the terminal ileum, ${ }^{18}$ and in a study, SIBO and $C$. difficile colonization as the terminal ileal microbiota has been evaluated. ${ }^{19}$ To date, microbiota changes in terminal ileum with PPI administration could be summarized as an increase of Firmicutes (Lactobacillus or Clostridium) and/or a decrease of Bacteroidetes. ${ }^{9}$ However, changes of gut microbiome by PPI depend on the species and intestinal sampling site. In dogs, administration of high-dose PPIs increased Lactobacillus but decreased Bacteroidetes in feces. ${ }^{20}$ In Wistar rats, there were significant 
increases in the fecal Lactobacillus levels without comparable increases in Bacteroidetes by PPI. ${ }^{21}$ In humans, the coadministration of antibiotics and PPIs caused reduced fecal bacterial diversity with decreased Bacteroidetes, which might predispose to $C$. difficile infection. ${ }^{22}$ In this study, terminal ileal luminal microbiota in lansoprazole-treated rats was quite distinct from those in controls, which was attributed to the predominance of Firmicutes in PPI-administrated rats (Fig. 1). Abundance of Firmicutes phylum resulted from an increased ratio of Clostridium g4 genus in three of six rats, but one rat showed a marked increase of Lactobacillus genus without an increase of Clostridium (Fig. 3).

With respect to the weight change, previous mouse and human studies have associated Firmicutes with obesity and Bacteroidetes with weight loss. ${ }^{23,24}$ In addition, weight loss is significantly associated with a decrease in the F/B ratio after Roux-en-Y gastric bypass surgery. ${ }^{25}$ Recently, one study have shown that perioperative PPI use was associated with an increase in Firmicutes and a decrease in Bacteroidetes in severely obese patients before and after laparoscopic Roux-en-Y gastric bypass surgery. ${ }^{26}$ The changes in bacterial composition in this study were similar to our results, with an increase of Firmicutes evident in the PPI-treated group. In this study, however, postoperative PPI use produced poorer weight loss than non-PPI use. The discrepancy in weight change could be explained in part by interspecies difference (humans vs F344 rats) and the difference in the origin of sample (fecal vs terminal ileal luminal microbiota). Furthermore, a recent study reported alterations in the gut microbiota (decrease in Akkermansia and Coprococcus and increase in Bacteroidales) and weight loss after administration of omeprazole in C57BL/6J mice. ${ }^{27}$ Therefore, the interactions between PPI and gut microbiota changes and weight loss may be more complex and might be influenced by other factors.

Generally, PPI causes the increase in both the quantity and diversity of the gastric microbiome, which causes an increase in the quantity of bacteria in the intestine, SIBO. ${ }^{9}$ In the present study there was no significant finding on the diversity of the bacterial community despite a tendency of increased OTU counts in the PPI-treated group (Supplementary Table 1). It might be attributed to a small sample size. However, a recent study reported diminishing observed OTU counts during PPI therapy, which was reversible after cessation of PPI usage within 1 month. ${ }^{28}$ To date, there is limited evidence, and further studies are needed.

There are some limitations of this study. The sample size was relatively small. Lansoprazole-treated rats were fed with ad libitum rat chow with PPI, and the daily dose taken each day could not be quantified. Weight gain or loss might be attributed to the potential differences in food intake. There was no body weight data mainly because it was a pilot study evaluating the change of small intestinal microbiota and we did not expect body weight change following the long-term PPI treatment. However, control and treatment experiments conducted simultaneously and there was no significant weight difference between the two groups. Finally, only luminal microbiota from terminal ileum was analyzed. Most studies investigated the changes in fecal microbiota, thus our findings may not be comparable to the previous studies. In addition, mucosa-associated microbiota is distinct from luminal microbial communities. ${ }^{29}$ However, it is still uncertain whether chronic PPI administration can alter luminal microbiota or mucosa-associated microbiota or both.

Further studies are on the way examining the different microbiota profiles between luminal microbiota from terminal ileum, mucosa-associated microbiota from cecum, and fecal microbiota. The use of aged rats is very difficult and time-consuming.

Despite these limitations, the present pilot study offers some valuable insights. The long-term administration of PPI may cause changes of microbiota in the terminal ileum and weight loss in F344 rats. However, this does not mean that the longterm PPI use also causes weight loss and a similar gut microbiota change in humans. In addition, PPIs are a mainstay in the treatment of various upper gastrointestinal disorders, but the safety of their long-term use is still uncertain. This pilot study does not confirm that the changes of gut microbiota are harmful. Therefore, further studies are warranted in this issue, especially in relation to SIBO or C. difficile infection.

\section{CONFLICTS OF INTEREST}

No potential conflict of interest relevant to this article was reported.

\section{ACKNOWLEDGEMENTS}

This work was supported by grant number 13-2015-002 from the Seoul National University Bundang Hospital Research fund.

\section{REFERENCES}

1. Pham CQ, Regal RE, Bostwick TR, Knauf KS. Acid suppressive therapy use on an inpatient internal medicine service. Ann Pharmacother 2006;40:1261-1266.

2. Ramirez E, Lei SH, Borobia AM, et al. Overuse of PPIs in patients at admission, during treatment, and at discharge in a tertiary Spanish hospital. Curr Clin Pharmacol 2010;5:288-297.

3. Reimer C. Safety of long-term PPI therapy. Best Pract Res Clin Gastroenterol 2013;27:443-454.

4. Jo Y, Park E, Ahn SB, et al. A proton pump inhibitor's effect on bone metabolism mediated by osteoclast action in old age: a prospective randomized study. Gut Liver 2015;9:607-614.

5. Atkinson NS, Reynolds DJ, Travis SP. 'Lemonade legs': why do some patients get profound hypomagnesaemia on proton-pump inhibitors? Intest Res 2015;13:227-232.

6. Lo WK, Chan WW. Proton pump inhibitor use and the risk of 
small intestinal bacterial overgrowth: a meta-analysis. Clin Gastroenterol Hepatol 2013;11:483-490.

7. Ratuapli SK, Ellington TG, O'Neill MT, et al. Proton pump inhibitor therapy use does not predispose to small intestinal bacterial overgrowth. Am J Gastroenterol 2012;107:730-735.

8. Husebye E, Skar V, Høverstad T, Iversen T, Melby K. Abnormal intestinal motor patterns explain enteric colonization with gramnegative bacilli in late radiation enteropathy. Gastroenterology 1995;109:1078-1089.

9. Freedberg DE, Lebwohl B, Abrams JA. The impact of proton pump inhibitors on the human gastrointestinal microbiome. Clin Lab Med 2014;34:771-785.

10. Jo HJ, Kim N, Nam RH, et al. The effect of Cochinchina momordica seed extract on gastric acid secretion and morphologic change in aged rat stomach. Gut Liver 2013;7:560-568.

11. Huber T, Faulkner G, Hugenholtz P. Bellerophon: a program to detect chimeric sequences in multiple sequence alignments. Bioinformatics 2004;20:2317-2319.

12. Kim OS, Cho YJ, Lee K, et al. Introducing EzTaxon-e: a prokaryotic 16S rRNA gene sequence database with phylotypes that represent uncultured species. Int J Syst Evol Microbiol 2012;62(Pt 3):716-721.

13. Li XX, Wong GL, To KF, et al. Bacterial microbiota profiling in gastritis without Helicobacter pylori infection or non-steroidal anti-inflammatory drug use. PLoS One 2009;4:e7985.

14. Gan KH, Geus WP, Lamers CB, Heijerman HG. Effect of omeprazole $40 \mathrm{mg}$ once daily on intraduodenal and intragastric $\mathrm{pH}$ in $\mathrm{H}$. pylori-negative healthy subjects. Dig Dis Sci 1997;42:2304-2309.

15. Hoffmann JC, Zeitz M. Small bowel disease in the elderly: diarrhoea and malabsorption. Best Pract Res Clin Gastroenterol 2002;16:17-36.

16. Husebye E, Skar V, Høverstad T, Melby K. Fasting hypochlorhydria with gram positive gastric flora is highly prevalent in healthy old people. Gut 1992;33:1331-1337.

17. Mariat D, Firmesse 0, Levenez F, et al. The Firmicutes/Bacteroidetes ratio of the human microbiota changes with age. BMC Microbiol 2009;9:123.

18. Pimentel M, Chatterjee S, Chang C, et al. A new rat model links two contemporary theories in irritable bowel syndrome. Dig Dis
Sci 2008;53:982-989.

19. Gerritsen J, Timmerman HM, Fuentes S, et al. Correlation between protection against sepsis by probiotic therapy and stimulation of a novel bacterial phylotype. Appl Environ Microbiol 2011;77:77497756.

20. Garcia-Mazcorro JF, Suchodolski JS, Jones KR, et al. Effect of the proton pump inhibitor omeprazole on the gastrointestinal bacterial microbiota of healthy dogs. FEMS Microbiol Ecol 2012;80:624636.

21. Kanno T, Matsuki T, Oka M, et al. Gastric acid reduction leads to an alteration in lower intestinal microflora. Biochem Biophys Res Commun 2009;381:666-670.

22. Jakobsson HE, Jernberg C, Andersson AF, Sjölund-Karlsson M, Jansson JK, Engstrand L. Short-term antibiotic treatment has differing long-term impacts on the human throat and gut microbiome. PLoS One 2010;5:e9836.

23. Ley RE, Bäckhed F, Turnbaugh P, Lozupone CA, Knight RD, Gordon JI. Obesity alters gut microbial ecology. Proc Natl Acad Sci U S A 2005;102:11070-11075.

24. Ley RE, Turnbaugh PJ, Klein S, Gordon J. Microbial ecology: human gut microbes associated with obesity. Nature 2006;444:10221023.

25. Sweeney TE, Morton JM. The human gut microbiome: a review of the effect of obesity and surgically induced weight loss. JAMA Surg 2013;148:563-569.

26. Ward EK, Schuster DP, Stowers KH, et al. The effect of PPI use on human gut microbiota and weight loss in patients undergoing laparoscopic Roux-en-Y gastric bypass. Obes Surg 2014;24:15671571.

27. Sands SA, Tsau S, Yankee TM, Parker BL, Ericsson AC, LeVine $\mathrm{SM}$. The effect of omeprazole on the development of experimental autoimmune encephalomyelitis in C57BL/6J and SJL/J mice. BMC Res Notes 2014;7:605.

28. Seto CT, Jeraldo P, Orenstein R, Chia N, DiBaise JK. Prolonged use of a proton pump inhibitor reduces microbial diversity: implications for Clostridium difficile susceptibility. Microbiome 2014;2:42.

29. Parkes GC, Brostoff J, Whelan K, Sanderson JD. Gastrointestinal microbiota in irritable bowel syndrome: their role in its pathogenesis and treatment. Am J Gastroenterol 2008;103:1557-1567. 